\title{
Anti-CD3 x EGFR bispecific antibody redirects cytokine-induced killer cells to glioblastoma in vitro and in vivo
}

\author{
PAN MA ${ }^{1,2^{*}}$, QIANG HE ${ }^{3 *}$, WEI LI ${ }^{1}$, XIANLIANG $\mathrm{LI}^{3}$, HUAMIN HAN $^{1}$, MENGMENG JIN $^{4}$,

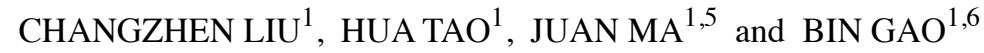 \\ ${ }^{1}$ CAS Key Laboratory of Pathogenic Microbiology and Immunology, Institute of Microbiology, Chinese Academy of \\ Sciences; ${ }^{2}$ Graduate University of Chinese Academy of Sciences, Beijing 100101; ${ }^{3}$ The Beijing Chao-Yang Hospital \\ Capital Medical University, Beijing 100020; ${ }^{4}$ The General Hospital of Chinese People's Liberation Army, Beijing 100853; \\ ${ }^{5}$ Beijing Shijitan Hospital, Capital Medical University, Beijing 100038; ${ }^{6}$ China-Japan Joint Laboratory of Molecular \\ Immunology and Microbiology, Institute of Microbiology, Chinese Academy of Sciences, Beijing 100101, P.R. China
}

Received March 17, 2015; Accepted May 14, 2015

DOI: 10.3892/or.2015.4233

\begin{abstract}
The epidermal growth factor receptor (EGFR) is an attractive target for the immunotherapy of $\mathrm{EGFR}^{+}$tumors. Adjuvant immunotherapy with cytokine-induced killer (CIK) cells may improve progression-free survival rates in patients suffering from cancer. In the present study, we examined the bispecific antibody anti-CD3 $\mathrm{x}$ anti-EGFR (EGFRBi-Ab) for its ability to redirect CIK cells to target EGFR-positive glioblastoma. The specific cytolytic activity of CIK cells armed with EGFRBi-Ab against U87MG-luc cells was evaluated by bioluminescent signal generated using luciferase reporter assay which did not alter the surface molecule expression or proliferation ability of U87MG cells. In contrast to unarmed CIK cells, increased cytotoxic activity of EGFRBi-armed CIK cells against the U87MG-luc target was observed at effector/ target (E/T) ratios of 5:1, 10:1, and 20:1. Moreover, EGFRBiarmed CIK cells secreted significantly higher levels of IFN- $\gamma$, TNF- $\alpha$, and IL-2 than their unarmed CIK counterpart cells.
\end{abstract}

Correspondence to: Dr Juan Ma or Professor Bin Gao, CAS Key Laboratory of Pathogenic Microbiology and Immunology, Centre for Molecular Immunology, Institute of Microbiology, Chinese Academy of Sciences, 1 Beichen West Road, Beijing 100101, P.R. China

E-mail: majuan96@sina.com

E-mail: bgao2004@gmail.com

*Contributed equally

Abbreviations: ANOVA, one-way analysis of variance; GBM, glioblastoma; CIK, cytokine-induced killer; EGFR, epidermal growth factor receptor; EGFRBi-Ab, anti-CD3 x anti-EGFR bispecific antibody; PBMCs, peripheral mononuclear blood cells; TAA, tumorassociated antigen

Key words: immunotherapy, EGFR, bispecific antibody, luciferase, glioblastoma
Furthermore, in glioblastoma xenograft mice, infusion of the EGFRBi-armed CIK cells successfully inhibited the growth of glioblastoma tumors. The in vitro and in vivo antitumor effects of EGFRBi-armed CIK cells support their clinical use for treatment of glioblastoma in the future.

\section{Introduction}

Glioblastoma (GBM) is the most common primary malignancy in brain. The Central Brain Tumor Registry of the United States reports the annual incidence of GBM at $\sim 3.19 / 100,000$ people with a median survival of 15 months (1). The commonly used strategies for treatment include surgery, radiation, and chemotherapy. Although chemotherapy modestly increases survival in patients when surgery and radiotherapy are unsuccessful, the prognosis of GBM is primarily poor (2). Cancer immunotherapy recognized as the fourth antitumor modality has undergone a period of growth following encouraging data regarding its clinical efficacy (3). Immunotherapy approaches are under investigation for GBM, which target the following aspects: enhancing immune response to tumors, inhibiting or destroying molecular or cellular immunosuppressive mediators induced by GBM cells, generating monoclonal antibodies targeting the special tumor antigen in order to eliminate tumor cells, and in vitro expanding tumor-specific lymphocytes naturally arising or manually modified (4). The current overall therapeutic outcomes of GBM are encouraging.

GBM is frequently associated with epidermal growth factor receptor (EGFR) overexpression, and EGFR signaling is important in various types of cancer, including GBM (5). Although noteworthy results of antibody-based therapy in the treatment of melanoma, renal cell carcinoma, and hematologic cancers have been observed, the treatment has not directly benefitted GBM patients (6-8). The antibody specific for EGFR, Erbitux ${ }^{\circledR}$, showed little effect on GBM patients (9). In early-stage clinical trials, studies have shown promising results for the use of bispecific antibody targeting CD3 and glioma antigen (10-13). Of note, $\mathrm{CD} 8^{+} \mathrm{T}$-cell infiltrate is associated with prolonged survival of newly diagnosed GBM patients (14). Additionally, almost half of the T cells 
infiltrating GBM specimens were CD56 ${ }^{+} \mathrm{T}$ cells (15), and antiCD3 $\mathrm{x}$ anti-GD2 bispecific antibody was able to redirect T-cell cytolytic activity to a neuroblastoma target (16). The abovementioned observations suggest that adoptive immunotherapy for GBM mediated by bispecific antibodies redirected effector lymphocyte is a promising treatment.

To assess the efficiency of the antitumor effect of the bispecific antibody, a reliable sensitive mouse model is needed. The bioluminescence living imaging system as a novel image measuring technology, has been used widely due to its high sensitivity and accuracy in the detection of tumor growth (17). In the present study, we constructed a U87MG-luc cell line that expressed luciferase stably, and a linear correlation was identified between bioluminescent signal intensity and the number of U87MG-luc cells in vitro and in vivo. Clinically approved antiCD3 antibody was then chemically conjugated with Erbitux ${ }^{\circledR}$. Considering adjuvant immunotherapy with cytokine-induced killer (CIK) cells can improve progression-free survival rates (18), combination treatments may further improve the survival rates. Thus, the anti-CD3 $\mathrm{x}$ anti-EGFR bispecific antibody (EGFRBi-Ab) was used to direct CIK cells to kill the GBM target. Redirected with EGFRBi-Ab, CIK cells exhibited enhanced specific cytotoxicity and cytokine production ability. The efficacy of EGFRBi-Ab-armed CIK cells for the inhibition of EGFR-positive GBM tumor was also investigated in a SCID-Beige mouse model.

\section{Materials and methods}

Cell lines and vector construction. Human U87MG glioblastoma were obtained from the American Type Culture Collection (ATCC; Rockville, MD, USA). Colo205-luc, HT-29-luc, A549-luc, NCIH460-luc, BXPC-3-luc, MDA-MB231-luc, HeLa-luc and PC-3M-luc cell lines were purchased from Caliper Life Sciences (Hopkinton, MA, USA). Agents for cell culture were from Gibco-Life Technologies (Carlsbad, CA, USA).

The 3.71n-luc2 plasmid (previously constructed by our laboratory) contained a BirA substrate peptide and c-myc tag linked to a truncated membrane-anchored human low affinity nerve growth factor receptor ( $\triangle \mathrm{LNGFR}$ ). The 3.7BirA plasmid (also previously constructed by our laboratory) contained an ER retention signal and BirA enzyme as a reported gene. BirA substrate peptide transported to the cell surface was biotinylated by BirA enzyme to label the target cells (19).

Generation of the U87MG-luc reporter cell line. The 3.7lnluc2 and 3.7BirA lentiviral transfer vectors are selfinactivating lentivectors used for the separation of the target luciferase reported cells. VSV-G pseudotyped lentiviral vectors stocks were prepared by Lipofectamine 2000 transfection reagent-based transfection of 293T cells with $6 \mu \mathrm{g}$ of packaging plasmids pLP1, pLP2 and pLP/VSVG (Invitrogen, Carlsbad, CA, USA), respectively, and $3 \mu \mathrm{g}$ of transfer vector, respectively. The supernatant collected was filtered through $0.45-\mu \mathrm{m}$ filter and stored at $-80^{\circ} \mathrm{C}$. U87MG $\left(1 \times 10^{5}\right)$ cells seeded in a 6-well tissue culture plate 1 day in advance were infected with the viruses at a MOI of 1 by spin inoculation in a centrifuge at $1,800 \mathrm{x} \mathrm{g}$ for $90 \mathrm{~min}$ at $32^{\circ} \mathrm{C}$ with $8 \mu \mathrm{g} /$ $\mathrm{ml}$ polybrene. Magnetic beads-based cell separation was followed $72 \mathrm{~h}$ after post-infection according to the manufac- turer's instrucitons (19). After infection and separation, target luciferase-labeled cells were cultured in a black 96-well plate by limiting dilution. The transduction efficiency and expressed protein tag were monitored by flow cytometry. The bioluminescent imaging signals of selected single-clone U87MG-luc reporter cell lines were measured using the IVIS lumina system (Caliper Life Sciences) in a 96-well plate in culture medium with D-luciferin substrate at a final concentration of 0.15 mg/ml (Bc219-05; Synchem Chemie, Kassel, Germany).

In vitro cell proliferation. The U87MG and U87MG-luc cells were seeded in a 96 -well plate and incubated at $37^{\circ} \mathrm{C}$ overnight. One hundred microliters of fresh medium containing $10 \mu \mathrm{l}$ Cell Counting Kit-8 (CCK-8; Dojindo Laboratories, Kumamoto, Japan) was added to each well and incubated for an additional $1 \mathrm{~h}$. The absorbency of U87MG and U87MG-luc cells was measured at $450 \mathrm{~nm}$ by a 96-well plate reader (DG5032; Huadong, Nanjing, China) after incubation. According to the manufacturer's instructions, the proliferation of U87MG and U87MG-luc cells was assessed by the absorbance values.

FlowJocytometric analysis. Anti-CD3-FITC, anti-CD56-APC, anti-human B7-H1-PE and anti-mouse IgG2a-FITC secondary antibodies were purchased from eBioscience (San Diego, CA, USA). Anti-human B7-H3-PE was purchased from R\&D System (Minneapolis, MN, USA), anti-human GD2 was purchased from Millipore (Billerica, MA, USA) and anti-human IgG Fc-PE secondary antibody was purchased from BioLegend (San Diego, CA, USA). The cells were assayed with a Guava flow cytometer EasyCyte (Guava Technologies, Hayward, CA, USA) and the data analysis was carried out with the FlowJo software version 7.6.1 (Tree Star Inc., Ashland, OR, USA).

Preparation of CIK cells from peripheral blood lymphocytes (PBMCs). Peripheral mononuclear blood cells (PBMCs) were separated by Ficoll density gradient centrifugation. Blood was obtained from healthy donors as supplied by the Beijing Blood Bank. CIK cells were expanded 15 days from PBMCs as previously described (20). Briefly, the CIK cells were stimulated by combination of IFN- $\gamma$, interleukin- $1 \alpha$, interleukin-2 (Peprotech, Rocky Hill, NJ, USA) and anti$\mathrm{CD} 3 \mathrm{mAb}$ (OKT3; eBioscience). Fresh medium containing fresh interleukin-2 was added every 2 or 3 days and the cells were cultured for 15 days prior to being cryopreserved. The study was performed according to the protocols approved by the Biomedical Research Ethics Committee of CAS Key Laboratory of Pathogenic Microbiology and Immunology.

Synthesis of anti-CD3 $x$ anti-EGFR bispecific antibody (EGFRBi-Ab) and arming of CIK cells. Anti-EGFR (Erbitux ${ }^{\circledR}$; Merck Serono, Darmstadt, Germany) or anti-HER2 (Herceptin ${ }^{\circledR}$; Roche, Indianapolis, IN, USA) was reacted with sulfo-SMCC and anti-CD3 (OKT3) was reacted with Traut's reagents as previously described $(21,22)$. Cryopreserved CIK cells were thawed, and armed with EGFRBi-Ab at a concentration of $50 \mathrm{ng} / 10^{6}$ cells at room temperature for 30 min followed by washing the cells to eliminate unbound antibodies. The combination of OKT3 $\left(50 \mathrm{ng} / 10^{6}\right.$ cells $)$ and Erbitux $^{\circledR}\left(50 \mathrm{ng} / 10^{6}\right.$ cells) pre-incubated CIK cells were used as unarmed control CIK cells. 
A 3.7EF-1 $\alpha$-ER- DLNGFR-CMV-Luciferase

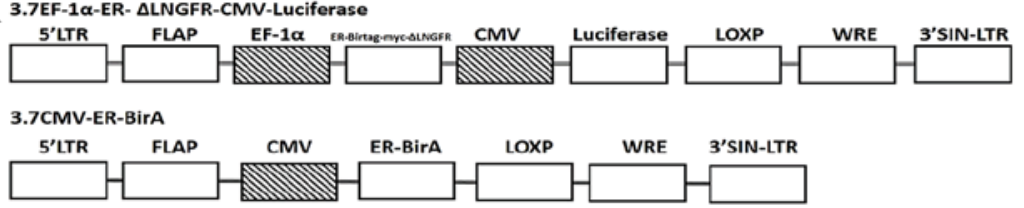

B

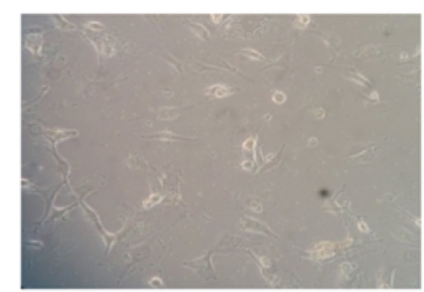

C Cell number of U87MG-luc cell

$\begin{array}{lllllllll}0 & 1562 & 3125 & 6250 & 12500 & 25000 & 50000 & 100000\end{array}$
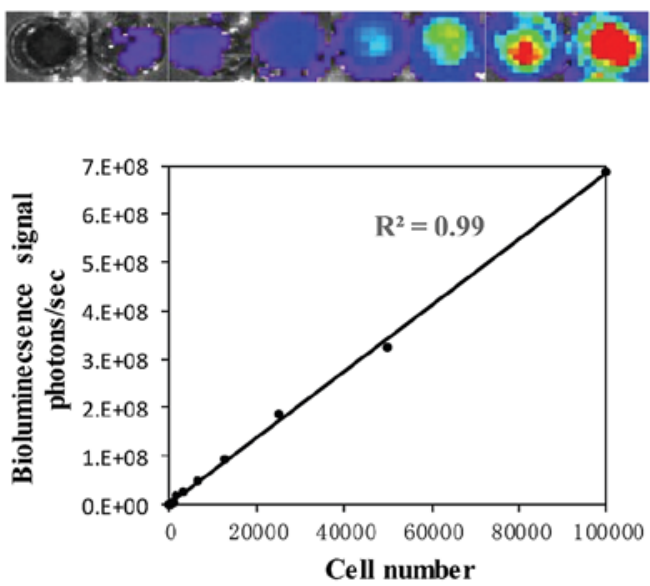

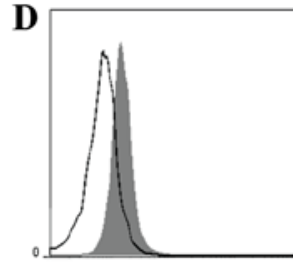

Anti-myc

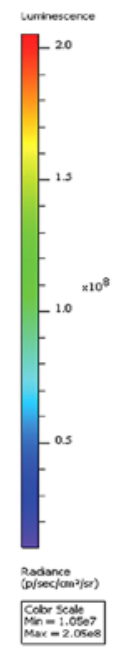

E

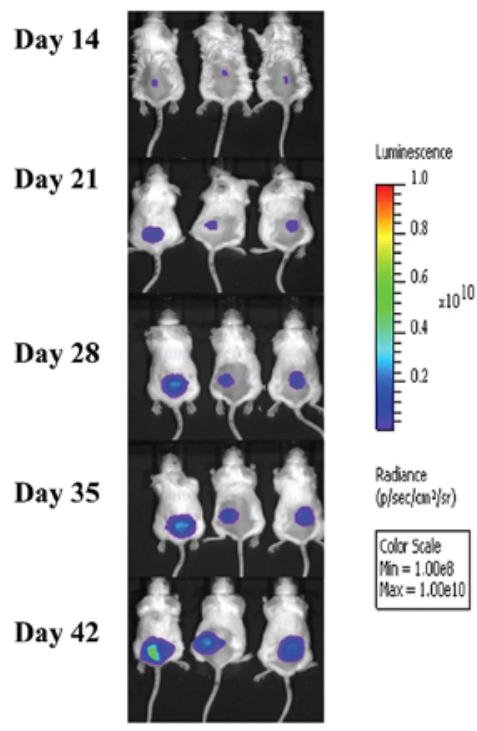

F

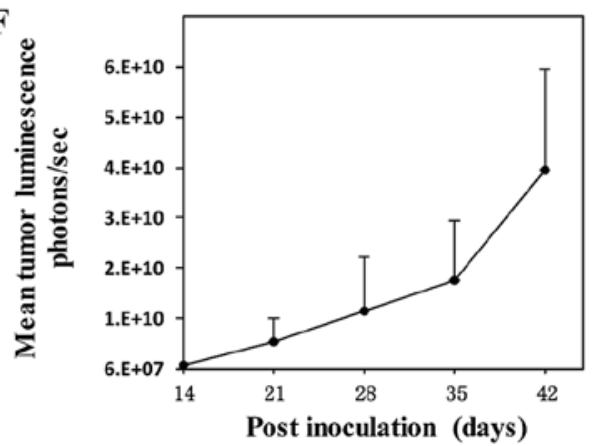

Figure 1. Construction of plasmid and establishment of U87MG-luc cells. (A) Diagrammatic structure of two lentiviral vectors: 3.71nluc2 and 3.7BirA. (B) U87MG cells infected with 3.7ln-luc2 and 3.7BirA lentivirus were isolated by DynaBeads, and positive cells were harvested. (C) The bioluminescence image signal was measured with the IVIS lumina system. Serial dilution of U87MG-luc cells was plated in a black 96-well plate in PBS containing D-luciferin substrate at a final concentration of $0.15 \mathrm{mg} / \mathrm{ml}$. Pearson's correlation coefficient $(\mathrm{R})$ was used to analyze between the luciferase quantity and living cell number is 0.99. (D) FACS analysis of c-Myc expression on selected U87MG-luc cells. (E) U87MG-luc cells $\left(5 \times 10^{6}\right)$ were injected subcutaneously into the dorsal region of female mice ( 6 weeks old, $\mathrm{n}=5$ ) and the bioluminescence signal was monitored by the in vivo imaging system at the indicated days after inoculation. (F) Mean tumor luminescence signal of inoculated SCID-Beige mice was measured by photons per second.

In vitro cytotoxicity assay. Cytotoxicity was measured with a luciferase quantitative assay (21-23). Target cells were seeded in triplicate in 96 -well microplates at $1 \times 10^{4} /$ well prior to the addition of EGFRBi-armed, Her2Bi-armed, or unarmed CIK cells at various effector-to-target $(\mathrm{E} / \mathrm{T})$ ratios. Effector and tumor cells were allowed to interact at $37^{\circ} \mathrm{C}$ for $18 \mathrm{~h}$. A final concentration of $0.15 \mathrm{mg} / \mathrm{ml} \mathrm{D}$-luciferin was added to each well. The IVIS lumina system was used to measure the bioluminescent image signal.

ELISA assay. U87MG-luc cells were seeded (1x10 $/$ well) in 96-well microplates in triplicate overnight. The medium was removed, and fresh medium or medium containing EGFRBi-armed CIK or control CIK cells was added to wells at an E/T of 20:1. The CIK and target U87MG-luc cells were incubated at $37^{\circ} \mathrm{C}$ for $18 \mathrm{~h}$. The supernatants were collected and production of IFN- $\gamma$, TNF- $\alpha$, and IL-2 was quantified by the human cytokine ELISA kit (eBioscience) according to the manufacturer's instructions.

In vivo antitumor effect of EGFR-BiAb-armed CIK cells. Female eight-week-old SCID-Beige mice were purchased from the Peking University Health Science Center (Beijing, China). The U87MG-luc cells $\left(5 \times 10^{6}\right)$ were injected subcutaneously into the dorsal region of female SCID-Beige mice. For the tumor growth inhibition studies, on the following day, EGFRBiAb-armed CIK cells $\left(5 \times 10^{7} / \mathrm{mouse}\right)$ or control CIK cells were injected intravenously. Tumor growth was monitored on the indicated day by the IVIS lumina imaging system with Living Image software. The signal intensity of tumor burdens was expressed as total photons $/ \mathrm{second} / \mathrm{cm}^{2}$ ( $\left.\mathrm{p} / \mathrm{s} / \mathrm{cm}^{2} / \mathrm{sr}\right)$.

Statistical analysis and reproducibility. Experiments were repeated at least three times. Data were analyzed using Graphpad Prism 5 software and were presented as the means \pm SDs. Unpaired Student's t-test (two-tailed) or the Mann-Whitney test was used for comparison of two groups where appropriate. One-way analysis of variance (ANOVA) followed by Dunnett's post hoc were used for multiple comparisons. Pearson's correlation coefficient (R) was analyzed. $\mathrm{P}<0.05$ was considered statistically significant. The number with a significant difference from a control is denoted by an asterisk in the figures. 

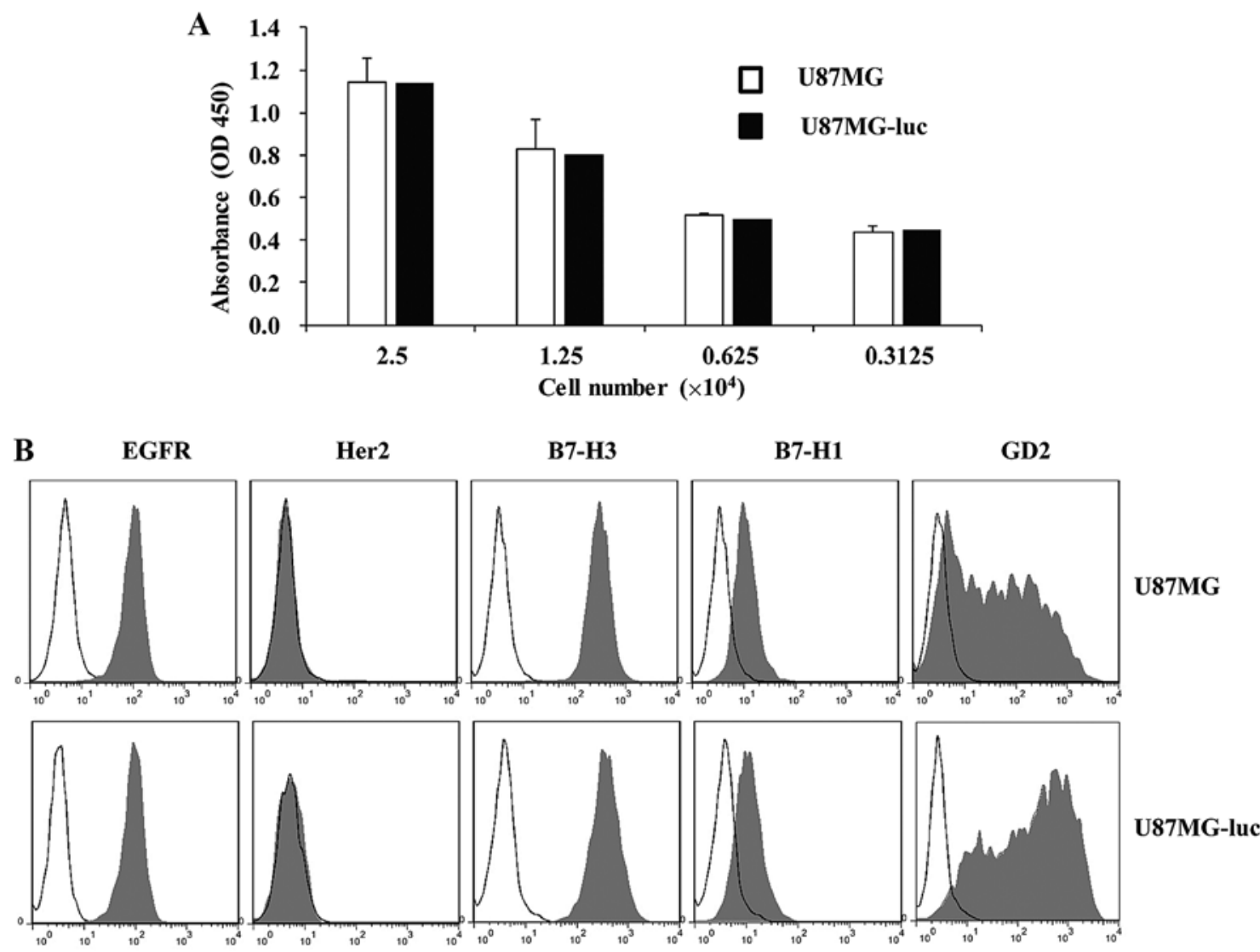

Figure 2. Luciferase gene has no effect on cell proliferation and surface molecular expression of U87MG cells. (A) Cell proliferation of U87MG and U87MG-luc cells was assessed with CCK- 8 assay. The experiments were performed in triplicate, and the mean absorbance of different cell numbers at 450 nm was shown as SD. Statistical analysis was conducted using the Student's t-test. (B) Surface expression of EGFR, Her2, B7-H3, B7-H1, and GD2 on U87MG cells and U87MG-luc was analyzed by flow cytometry. Shaded histogram shows cells stained with molecule-specific antibody, and open histogram shows cells stained with isotype control IgG. EGFR, epidermal growth factor receptor.

\section{Results}

Establishment of human U87MG-luc glioblastoma cell line stably expressing luciferase. To establish U87MG-luc cells, 3.7ln-luc2 and 3.7BirA lentivirus plasmids (Fig. 1A) were constructed and used to produce pseudotyped lentiviruses. The U87MG cells infected with the lentivirus were able to express luciferase protein and process the transmembrane protein on the surface of cells. After adding the substrate d-biotin, BirA-tag was biotinylated, attached to streptavidin beads, and positive cells were subsequently separated (Fig. 1B). A single U87MG-luc clone was obtained by serial limiting dilutions in 96-well plates and the luciferase expression was detected and analyzed by the IVIS lumina imaging system with Living Image software. We seeded a number of U87MG-luc cells in 96-well plates ranging from 1,562 to $100,000(1,562,3,125$, $6,250,12,500,25,000,50,000$ and 100,000) per well. The results showed that there was a good correlation between the level of luciferase activity and the number of U87MG-luc cells (Fig. 1C). The linear correlation between the luciferase activity-related bioluminescent signal and the number of cells is shown in Fig. 1C $\left(\mathrm{R}^{2}=0.99\right)$. Moreover, the expression of myc-BirA-tag was detected by FACS staining with anti-myc antibody (Fig. 1D).

To investigate whether U87MG-luc cells could be used to establish a subcutaneous tumor model in SCID-Beige mice,
$5 \times 10^{6}$ U87MG-luc cells were injected subcutaneously in the dorsal thigh of female mice $(n=6)$, and the bioluminescent signal reflecting tumor growth was monitored and analyzed using the IVIS lumina imaging system with Living Image software at the indicated days (Fig. 1E). Mean tumor luminescence was also calculated (Fig. 1F). Tumor size was measured and a linear correlation between mean luminescence and mean tumor size was observed $\left(\mathrm{R}^{2}=0.99\right.$, data not shown).

Luciferase gene has no effect on cell proliferation and surface molecular expression of U87MG cells. To investigate whether the luciferase gene itself had a negative effect on U87MG cell growth in vitro, proliferation assays were performed (Fig. 2A). To measure the proliferation of cells stably expressing luciferase, the proliferation rates of U87MG and U87MG-luc cells were detected by a CCK- 8 assay as described in Materials and methods. The results showed that there was no significant difference in proliferation between the U87MG and U87MG-luc cells.

We detected the expression of EGFR, Her2, B7-H3, B7-H1 and GD2, the attractive molecules used as the targets for glioblastoma, with the specific antibody on the surface of U87MG and U87MG-luc, respectively. As shown in Fig. 2B, a high expression of EGFR, B7-H3 and GD2 and a low expression of B7-H1 was detected on U87MG and U87MG-luc cells. By contrast, Her2 was not detected on the cells. Moreover, the 
A a
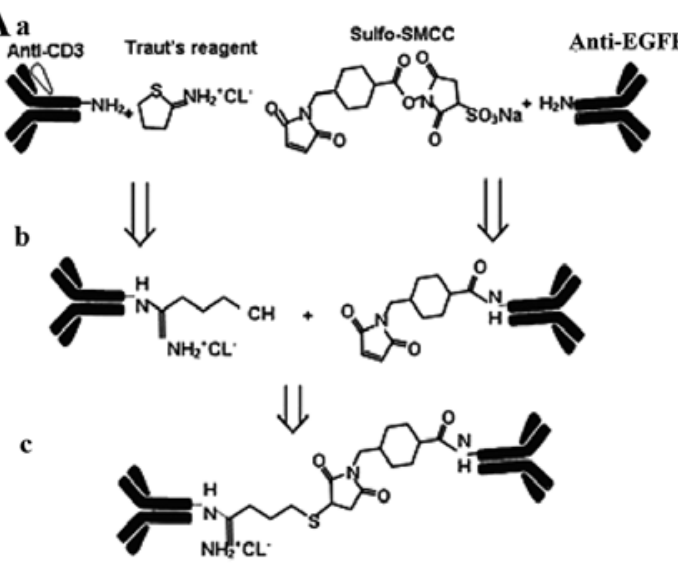

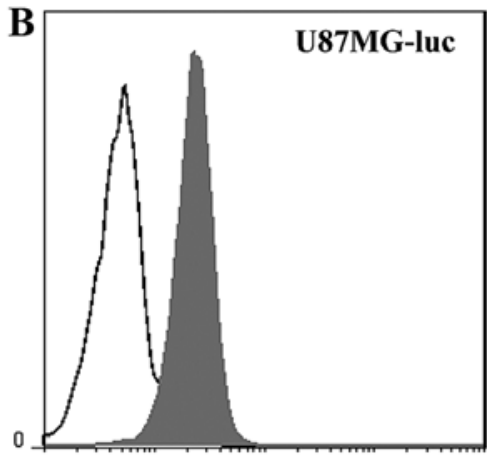

Anti-mouse IgG2a

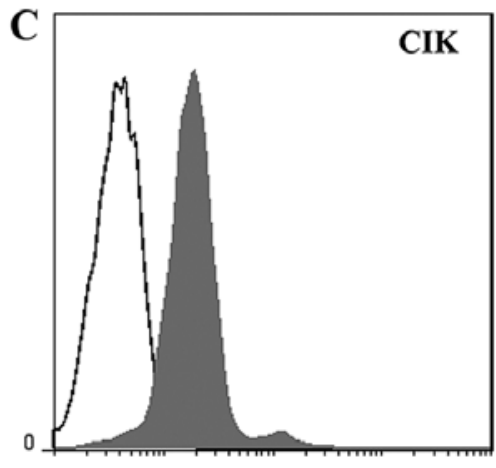

Anti-human IgG Fc
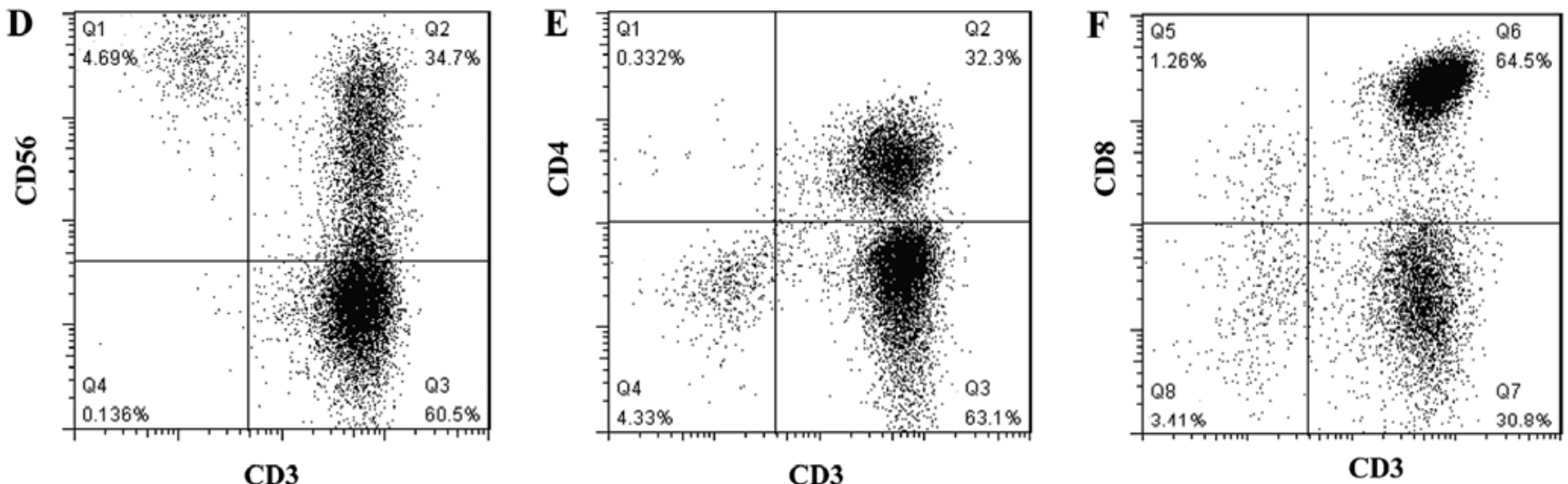

Figure 3. General scheme for the generation of anti-CD3 x anti-EGFR bispecific antibody and analysis of cytokine-induced killer cells. (A) General scheme for the generation of anti-CD3 $x$ anti-EGFR bispecific antibody (EGFRBi-Ab). (B) Flow cytometry-based binding assay for EGFRBi-Ab. U87MG-luc cells were incubated with EGFRBi-Ab $\left(1 \mu \mathrm{g} / \mathrm{ml}\right.$, shaded histogram) or a mixture of OKT3 and Erbitux ${ }^{\circledR}(1 \mu \mathrm{g} / \mathrm{ml}$, open histogram). EGFRBi-Ab binding was examined by detecting anti-CD3 moiety of the BiAb with FITC-labeled anti-mouse IgG2a. (C) CIK cells were incubated with EGFRBi-Ab (1 $\mu \mathrm{g} / \mathrm{ml}$, shaded histogram) or a combination of OKT3 and Erbitux ${ }^{\circledR}(1 \mu \mathrm{g} / \mathrm{ml}$, open histogram). The bound CD3 moiety of EGFRBi-Ab to CIK cells was assessed by detecting the EGFR moiety of BiAb stained with PE-labeled anti-human IgG Fc with flow cytometry. (D-F) Flow cytometric analysis of cell-specific surface molecule expression on CIK cells. Images shown are representative of one of at least five experiments. CIK, cytokine-induced killer.

expression level of these molecules was similar on the surface of U87MG and U87MG-luc cells. Taken together, these results indicated that the luciferase gene did not affect the proliferation or target the molecular expression of U87MG cells.

Therefore in the subsequent experiment, we selected one of the molecules with a high expression on U87MG-luc and EGFR, as the target for arming CIK cells.

Preparation and characterization of EGFRBi-Ab and CIK cells. Bispecific antibody EGFRBiAb was prepared as described in Materials and methods (Fig. 3A). Firstly, the binding specificity of EGFRBi against EGFR was assessed. U87MG-luc cells were stained with EGFRBi-Ab or a combination of OKT3 and Erbitux ${ }^{\circledR}$ (used as the unarmed control for EGFRBi-Ab). Anti-mouse IgG2a-FITC was then added to detect the CD3 moiety of EGFRBi-Ab. Only functionally bispecific EGFRBi antibody bound to U87MG-luc cells by EGFR-recognized Erbitux ${ }^{\circledR}$ and were detected through mouse origin OKT3 by anti-mouse IgG2a secondary antibody. As shown in Fig. 3B, positively stained cells were detected in $87 \%$ of the U87MG-luc population with a mean fluorescent intensity (MFI) of 24. On the other hand, the bound CD3 moiety of EGFRBi-Ab to CIK cells was assessed by staining with PE-labeled anti-human IgG Fc to detect the EGFR moiety of
BiAb. Positively stained cells were detected in $88 \%$ of the CIK cell population with an MFI of 33 (Fig. 3C).

To produce a sufficient number of effector cells, PBMCs from buffy coat were stimulated by the combination of IFN- $\gamma$, IL-1 $\alpha$, IL-2 with OKT 3 for 15 days as described in Methods and materials. The CIK cells were then quantitatively analyzed by FACS. As shown in Fig. 3D-F, the CIK cells contained almost $96.74 \pm 1.49 \%$ of $\mathrm{CD}^{+}$cells, i.e., $20.02 \pm 9.4 \%$ of $\mathrm{CD}^{+} \mathrm{CD}^{+} 6^{+}$ cells (Fig. 3D), $36.1 \pm 8.37 \%$ of $\mathrm{CD}^{+} \mathrm{CD}^{+}$(Fig. 3E), and $61.46 \pm 7.43 \%$ of $\mathrm{CD}^{+}{ }^{+} \mathrm{CD}^{+}$(Fig. 3F) approximately. For the CD3 population, the majority of cells were CD56-positive, $2.73 \pm 1.43 \%$ (Fig. 3D). Overall, these data suggested that the CIK cells mainly comprised T cells and NK T cells with a small population of NK cells.

Cytotoxity effects of EGFRBi-armed CIK cells with IFN- $\gamma$ $T N F-\alpha$ and IL-2 production on glioblastoma cells. The amount of EGFRBi-Ab required to arm CIK cells ranged from 5 to $500 \mathrm{ng} / 10^{6}$ cells, with $50 \mathrm{ng}$ and $500 \mathrm{ng} / 10^{6}$ cells showing a similar cytotoxicity. Therefore, we selected $50 \mathrm{ng} / 10^{6}$ cells as the concentration of EGFRBi-Ab for all the subsequent experiments, and CIK cells mixed with individual OKT3 and Erbitux $^{\circledR}$ were considered unarmed CIK controls. Cytotoxicity assays were performed at $\mathrm{E} / \mathrm{T}$ ratios of 5:1, 10:1 and 20:1 for 
A

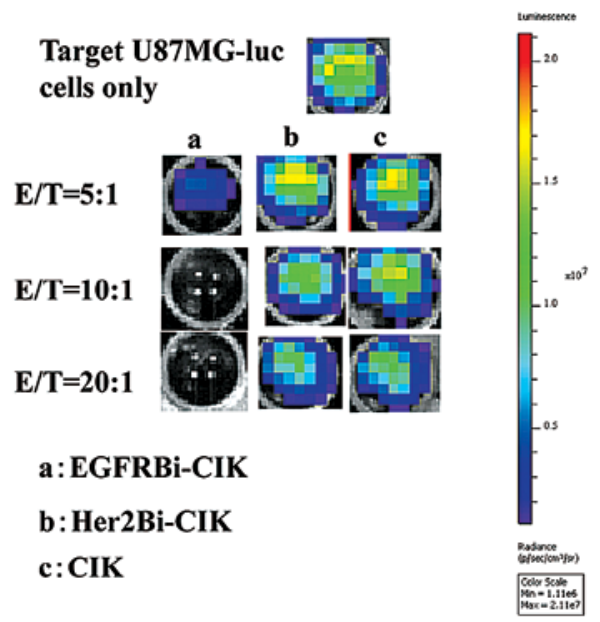

C IFN- $\mathbf{p}(\mathbf{p g} / \mathrm{ml})$

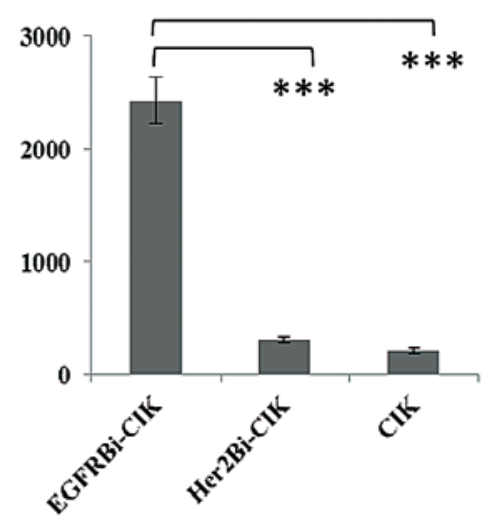

B

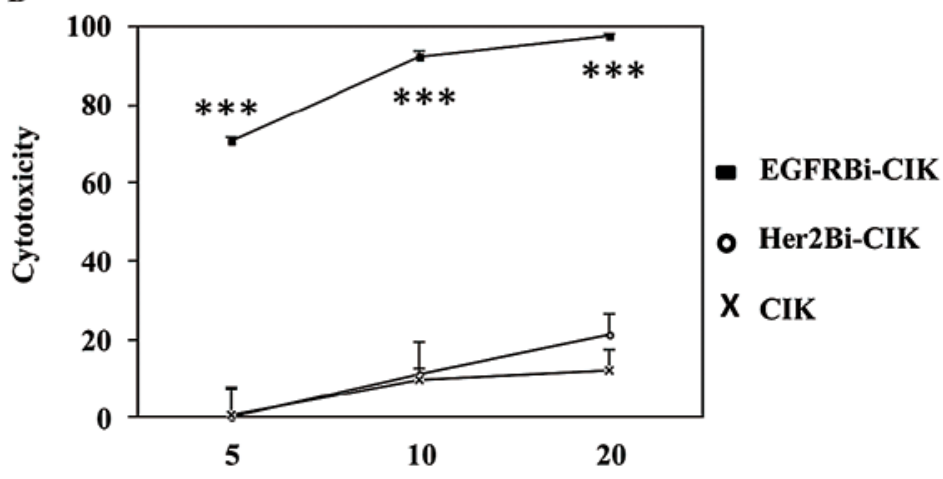

E:T
E $\quad$ IL-2 $(\mathrm{pg} / \mathrm{ml})$
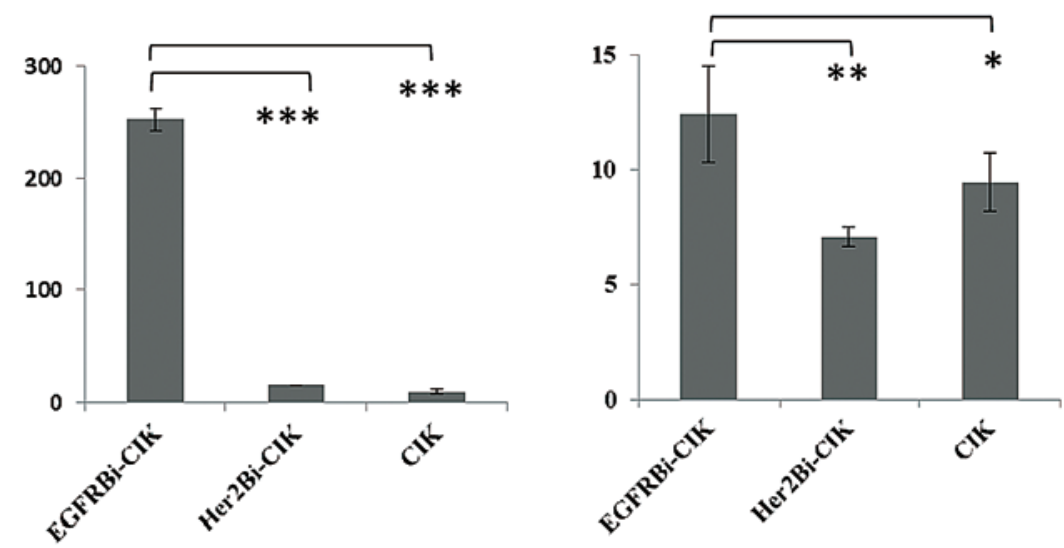

Figure 4. In vitro cytotoxicity assay mediated by EGFRBi-armed CIK against U87MG-luc cells (A) Bioluminescence images of U87MG-luc cells after incubation with EGFRBi-armed CIK or unarmed CIK cells or Her2Bi-armed CIK cells at different E/T ratio (5:1, 10:1 and 20:1) for 18 h. (B) Bioluminescence image signal in photons per second was converted into a living cell number and the cytotoxicity assay was measured at the indicated E/T. (C) IFN- $\gamma$, (D) TNF- $\alpha$ and (E) IL-2 secretion by EGFRBi-armed CIK cells against U87MG-luc cells. Supernatants of co-cultures at E/T of 20 were harvested at $18 \mathrm{~h}$ and analyzed for cytokine levels using a specific ELISA kit. Data are the mean $\pm \mathrm{SD}$ of triplicate determination. Image is a representative experiment of at least three experiments. "P<0.05, EGFRBi-armed CIK cells compared with control CIK cells under similar conditions. CIK, cytokine-induced killer; EGFR, epidermal growth factor receptor.

$18 \mathrm{~h}$. The bioluminescent images correlated with the number of living U87MG-luc cells (Fig. 4A). After 18-h incubation with EGFRBi-armed CIK or unarmed CIK or Her2Bi-armed CIK cells, bioluminescent image signal expressed in photons per second was converted into a living cell number and the cytotoxicity assays were calculated at the indicated $\mathrm{E} / \mathrm{T}$ ratios. As shown in Fig. 4B, an increasing E/T ratio was correlated directly with the percentage of cytotoxicity in all the tested CIK effectors. The percentage of cytotoxicity of EGFRBiarmed CIK cells was significantly greater than that of the other groups at each $\mathrm{E} / \mathrm{T}$ ratio.

To analyze the cytokines along with the cytotoxicity, supernatants of cell cultures were analyzed for cytokine production at an E/T of 20:1. As shown in Fig. 4C-E, significant increase was observed for IFN- $\gamma$, TNF- $\alpha$ and IL-2 secretion in EGFRBi-armed CIK cells over their unarmed CIK counterparts or Her2Bi-armed CIK cells when co-cultured with U87MG-luc cells.

Cytotoxity effects of EGFRBi-armed CIK cells on different tumor cell lines. We assessed the ability of EGFRBi-armed CIK cells to respond to a wide range of human EGFR-positive carcinoma, including colorectal (Colo205-luc and HT-29-luc), pancreatic (BXPC3-luc), lung (A549-luc and NCIH460-luc), breast (MDA-MB231-luc), cervical (HeLa-luc), and prostate (PC-3M-luc) cancer. After 18-h incubation with EGFRBi-armed CIK or unarmed CIK cells (Fig. 5), the percentage of cytotoxicity with EGFRBi-armed CIK cells was significantly greater than that with unarmed control effectors at E/T ratios of 5:1 and 10:1 in the EGFR-positive cancer cells.

EGFRBi-armed CIK cells inhibit U87MG tumor growth in SCID-Beige mice. To determine whether EGFRBi-armed CIK cells suppressed tumor growth in vivo, SCID-Beige mice were engrafted subcutaneously with U87MG-luc cells. On the following day, the mice were treated with unarmed control CIK cells or EGFRBi-CIK cells, respectively. After injection, the mice were given no further treatment but were monitored with bioluminescent imaging on the indicated day. This bioluminescent imaging model allows the monitoring of tumor cell fate as early as the first few days after inoculation, when tumor formation cannot be detected by palpation, and three representative mice of each group were shown (Fig. 6A). The mice treated with unarmed CIK cells showed stronger lumi- 

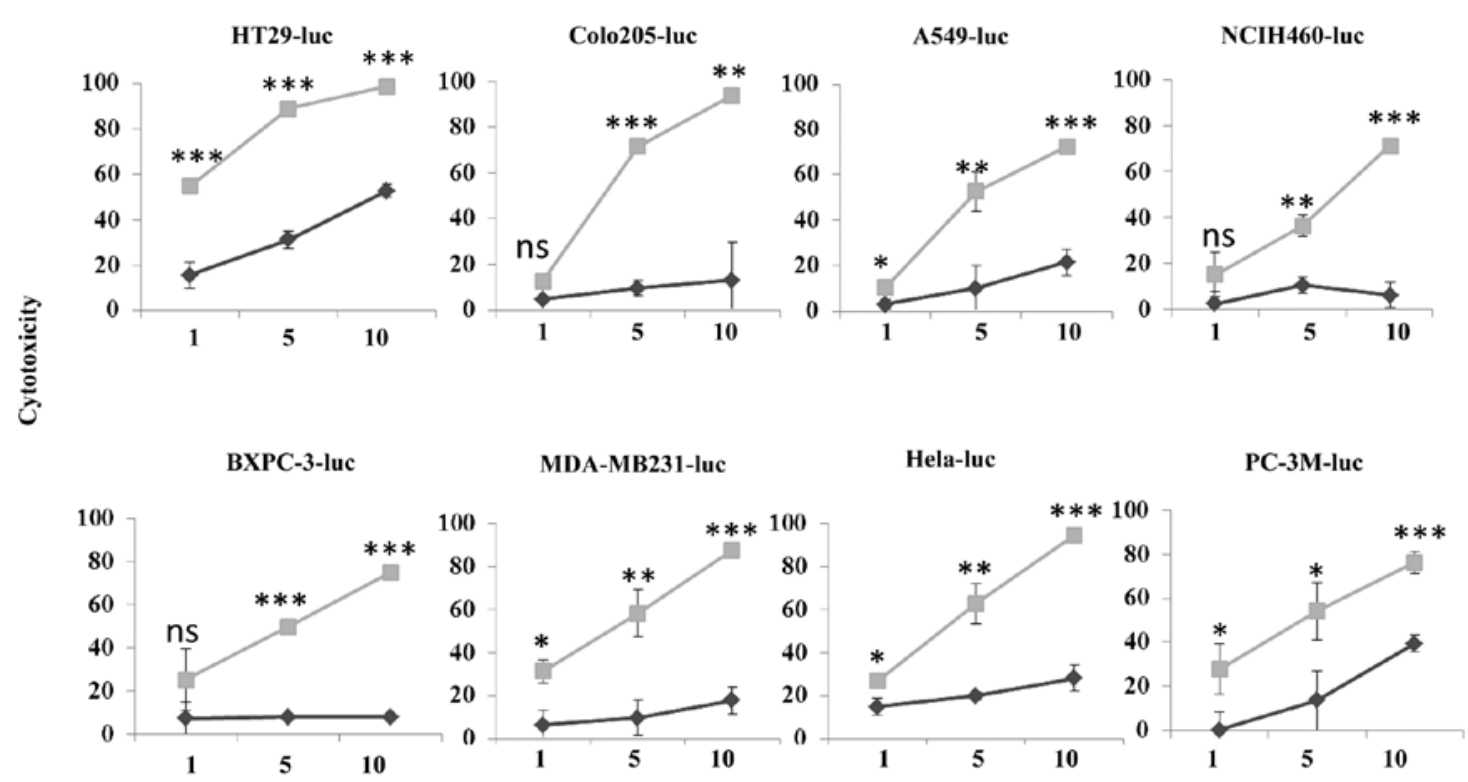

E:T

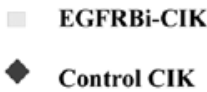

Figure 5. Antitumor effect of EGFRBi-armed CIK cells against different tumor cell lines. Target cells were incubated either with EGFRBi-armed CIK cells or a combination of OKT3 and Erbitux ${ }^{\circledast}$ with CIK cells (unarmed control CIK cells) for $18 \mathrm{~h}$. A luciferase quantitative assay was performed to determine cytotoxicity against different target cells at different $\mathrm{E} / \mathrm{T}$ ratios. The data are mean $\pm \mathrm{SD}$ of triplicate determination. Image is a representative experiment of at least three experiments. " $\mathrm{P}<0.05$, EGFRBi-armed CIK cells compared with unarmed CIK cells under similar conditions. CIK, cytokine-induced killer; EGFR, epidermal growth factor receptor.
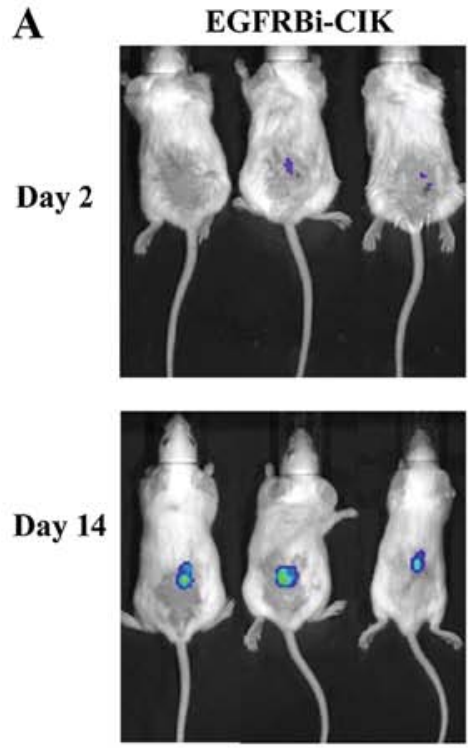
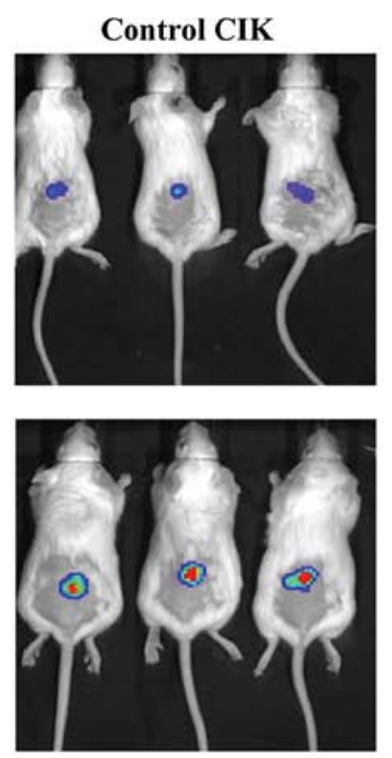

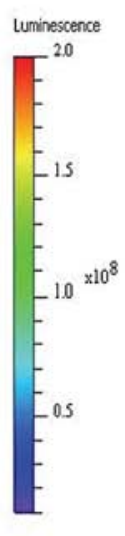

Radiance

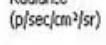
Color Scale
$\mathrm{Min}=1.00 \mathrm{e} 7$ $\operatorname{Mn}=1.00 \mathrm{e}$
$\operatorname{Max}=2.00 \mathrm{e} 8$
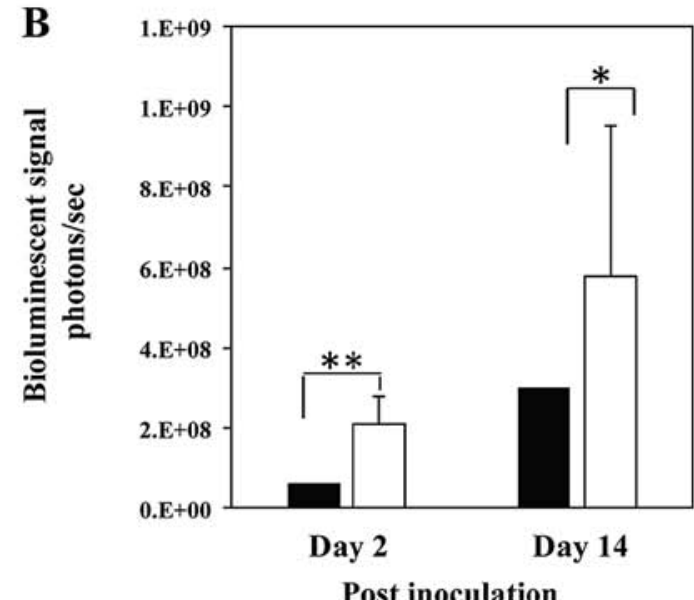

Post inoculation

EGFRBi-CIK Control CIK

Figure 6. In vivo cytotoxicity to U87MG-luc cells of EGFRBiAb-armed CIK cells in mouse model. (A) U87MG-luc cells $\left(5 \times 10^{6}\right)$ were injected subcutaneously into female SCID-Beige mice (6 weeks old, $\mathrm{n}=7$ per group). On the following day, EGFRBiAb-armed CIK cells (5x10 $/$ mouse) or unarmed control CIK cells were injected intravenously. In vivo bioluminescent signal was measured at the indicated days (day 2 and 14) using IVIS lumina imaging system. The signal intensity of tumor burdens was expressed as total photons $/ \mathrm{second} / \mathrm{cm}^{2}\left(\mathrm{p} / \mathrm{s} / \mathrm{cm}^{2} / \mathrm{sr}\right.$ ). (B) Mean bioluminescent imaging signal of mouse treated with EGFRBiAb-armed CIK or unarmed control CIK cells is shown at the indicated days. The significance between the two groups was compared by the Student's t-test. ( $\left.\mathrm{P}<0.05,{ }^{* *} \mathrm{P}<0.01\right)$. CIK, cytokine-induced killer; EGFR, epidermal growth factor receptor.

nescence than the EGFRBi-armed CIK cells. Furthermore, after comparing the mean luminescence of the two groups, significant differences in inhibition of tumor growth were observed between them (Fig. 6B). Therefore, compared with the unarmed control CIK cells, EGFRBi-armed CIK cells inhibited tumor growth in vivo. 


\section{Discussion}

Although the therapeutical antibody for EGFR, such as Erbitux ${ }^{\circledast}$, significantly improved survival rates in patients with metastatic colorectal cancer, these results could not been duplicated in GBM (9). Intratumor heterogeneity and EGFR pathway redundancy limited the clinical utility of the antibody-based therapy in GBM (24). Bispecific antibody comprises an immune effector cell-specific antibody and its hetero-conjugated $\mathrm{mAb}$ specific to a selected tumor-associated antigen (TAA). Such a bispecific antibody may redirect immune-potent effector cells to target tumor cells. EGFR is an ideal candidate used as a target in various types of tumor imaging and antibody-based therapeutic approaches. Preclinical findings have shown that arming activated $\mathrm{T}$ cells with bispecific EGFR antibody can target $\mathrm{EGFR}^{+}$cancers (25). In addition, adjuvant immunotherapy with CIK cells may prevent recurrence, and improve progression-free survival rates, and the quality of life (18). The combination of CIK cell therapy with conventional adjuvant or palliative therapies was superior to the standard therapy alone, indicating the benefit of CIK cell therapy for cancer patients (26). In our study, the CIK cells comprised T cells and NK T cells, as well as a small population of NK cells.

In the present study, we armed CIK cells with bispecific $\mathrm{Ab}$ and tested whether EGFR is a useful target for GBM. The results showed that, EGFRBi-armed CIK cells exhibited significant cytotoxic activity against human GBM U87MG cells in vitro. We also validated the specific lysis of a wide range of EGFR-positive human tumor cells, including lung, colorectal, pancreatic, breast, cervical, and prostate cancer by EGFRBi-Ab redirected CIK cells in vitro. Additionally, EGFRBi-armed CIK cells secreted a higher level of IFN- $\gamma$, TNF- $\alpha$, and IL-2 than unarmed CIK cells. It is conceivable that arming leads to binding specifically to tumor cells and the triggering of CIK cell activation and cytokine secretion. The increase in tumoricidal cytokines suggested that armed CIK cell infusions may vaccinate patients against their own tumors. Infusion of EGFRBi-armed CIK cells also markedly inhibited the growth of GBM cells in the xenograft mouse model.

The in vivo bioluminescence imaging (BLI) system has developed rapidly in recent years. With the sensitive, noninvasive, and quantitative system of BLI technology, it is possible to localize and monitor the orthotopic and metastatic growth of tumor in vivo (27). Specifically, BLI is widely used in cancer research and therapy (28). In the present study, we initially constructed a stable human U87MG-luc GBM cell line that expressed a high level of luciferase. As our data has shown, there was a good correlation between the luciferase activity and the number of cells. Furthermore, similar to the study by Tiffen et al (29), the luciferase gene did not affect the surface expression of EGFR, Her2, B7-H3, B7-H1, GD2 and the proliferation of GBM U87MG cells. We also established a xenograft subcutaneous human GBM tumor model in SCID-Beige mice with U87MG-luc cells and supplied a sensitive model for investigation of the pathogenesis of GBM, and assessment of the efficiency of immunotherapies.

In addition to adoptive cell therapy, the immune checkpoint blockade in passive immunotherapy has been successful in the treatment of various types of cancer, thereby encouraging a resurgence of interest in GBM (30). A new approach being evaluated in clinical trials involves the use of monoclonal antibodies to block immunosuppressive molecules such as PD-1 expressed by $\mathrm{T}$ cells. The monoclonal antibody specific for PD-1 is a promising treatment for GBM due to its tumor-expressed ligand PD-L1 (B7-H1), for predicting the efficacy of targeting the PD-1/PD-L1 pathway (31). In our study, administration of EGFRBi-armed CIK cells suppressed established tumor growth in vivo, although this did not completely eradicate the tumor cells. This occurred due to the insufficient persistence of armed-CIK cells. Function sustaining and trafficking of human CIK cells in the xenograft tumor model was more difficult than that of CIK cells in the immune system of the patient. In combination with anti-PD-1/PD-L1 antibody, anti-GD2 antibody or anti-B7-H3 antibody may further improve the in vivo efficacy of EGFRBi antibody-armed CIK cells (31-34). The combination of EGFRBi antibody- and GD2Bi antibody-armed CIK cells, and/or B7-H3Bi antibody-armed CIK cells is also a promising approach.

In summary, to the best of our knowledge, this study has shown for the first time that EGFRBi-Ab is capable of enhancing CIK cells ability to kill GBM and other EGFR-positive cancers. In addition, a sensitive model for evaluating antitumor effect for GBM has been generated. The in vitro and in vivo antitumor effect of EGFRBi-armed CIK cells supports their further clinical use for the treatment of GBM.

\section{Acknowledgements}

This present study is funded by the grants from the Ministry of Science and Technology of China (S\&T major program no. 2012ZX1004701-001-002), the Basic Research Program of China (973, no. 2013CB531502) and the National Nature Science Foundation of China (no. 31400754, 31370889, $81273270,81041110,81471590$ and 81402549$)$.

\section{References}

1. Thakkar JP, Dolecek TA, Horbinski C, Ostrom QT, Lightner DD, Barnholtz-Sloan JS and Villano JL: Epidemiologic and molecular prognostic review of glioblastoma. Cancer Epidemiol Biomarkers Prev 23: 1985-1996, 2014.

2. Stewart LA: Chemotherapy in adult high-grade glioma: A systematic review and meta-analysis of individual patient data from 12 randomised trials. Lancet 359: 1011-1018, 2002.

3. Mellman I, Coukos G and Dranoff G: Cancer immunotherapy comes of age. Nature 480: 480-489, 2011.

4. Agrawal NS, Miller R Jr, Lal R, Mahanti H, Dixon-Mah YN, DeCandio ML, Vandergrift WA III, Varma AK, Patel SJ, Banik NL, et al: Current studies of immunotherapy on glioblastoma. J Neurol Neurosurg 1: 21000104, 2014.

5. Bonavia R, Inda MM, Cavenee WK and Furnari FB: Heterogeneity maintenance in glioblastoma: A social network. Cancer Res 71: 4055-4060, 2011.

6. Gyorki DE, Spillane J, Speakman D, Shackleton M and Henderson MA: Current management of advanced melanoma: A transformed landscape. ANZ J Surg 84: 612-617, 2014.

7. Escudier B and Albiges L: Pazopanib for the treatment of advanced renal cell cancer. Expert Opin Orphan Drugs 2: 605-616, 2014

8. Capietto AH, Keirallah S, Gross E, Dauguet N, Laprévotte E, Jean C, Gertner-Dardenne J, Bezombes C, Quillet-Mary A, Poupot M, et al: Emerging concepts for the treatment of hematological malignancies with therapeutic monoclonal antibodies. Curr Drug Targets 11: 790-800, 2010.

9. Neyns B, Sadones J, Joosens E, Bouttens F, Verbeke L, Baurain JF, D'Hondt L, Strauven T, Chaskis C, In't Veld P, et al: Stratified phase II trial of cetuximab in patients with recurrent high-grade glioma. Ann Oncol 20: 1596-1603, 2009. 
10. Hishii M, Nitta T, Ebato M, Okumura K and Sato K: Targeting therapy for glioma by LAK cells coupled with bispecific antibodies. J Clin Neurosci 1: 261-265, 1994.

11. Jacobs SK, Wilson DJ, Melin G, Parham CW, Holcomb B, Kornblith PL and Grimm EA: Interleukin-2 and lymphokine activated killer (LAK) cells in the treatment of malignant glioma: Clinical and experimental studies. Neurol Res 8: 81-87, 1986.

12. Dillman RO, Duma CM, Schiltz PM, DePriest C, Ellis RA, Okamoto K, Beutel LD, De Leon C and Chico S: Intracavitary placement of autologous lymphokine-activated killer (LAK) cells after resection of recurrent glioblastoma. J Immunother 27: 398-404, 2004.

13. Pfosser A, Brandl M, Salih H, Grosse-Hovest L and Jung G: Role of target antigen in bispecific-antibody-mediated killing of human glioblastoma cells: A pre-clinical study. Int J Cancer 80: 612-616, 1999.

14. Yang I, Tihan T, Han SJ, Wrensch MR, Wiencke J, Sughrue ME and Parsa AT: $\mathrm{CD}^{+}{ }^{+}$T-cell infiltrate in newly diagnosed glioblastoma is associated with long-term survival. J Clin Neurosci 17: 1381-1385, 2010.

15. Waziri A, Killory B, Ogden AT III, Canoll P, Anderson RC, Kent SC, Anderson DE and Bruce JN: Preferential in situ $\mathrm{CD} 4^{+} \mathrm{CD} 56^{+} \mathrm{T}$ cell activation and expansion within human glioblastoma. J Immunol 180: 7673-7680, 2008.

16. Yankelevich M, Kondadasula SV, Thakur A, Buck S, Cheung NK and Lum LG: Anti-CD3 $x$ anti-GD2 bispecific antibody redirects T-cell cytolytic activity to neuroblastoma targets. Pediatr Blood Cancer 59: 1198-1205, 2012.

17. Henriquez NV, van Overveld PG, Que I, Buijs JT, Bachelier R, Kaijzel EL, Löwik CW, Clezardin P and van der Pluijm G: Advances in optical imaging and novel model systems for cancer metastasis research. Clin Exp Metastasis 24: 699-705, 2007.

18. Hontscha C, Borck Y,Zhou H, Messmer D and Schmidt-Wolf IG: Clinical trials on CIK cells: First report of the international registry on CIK cells (IRCC). J Cancer Res Clin Oncol 137: 305-310, 2011.

19. Han H, Liu Q, He W, Ong K, Liu X and Gao B: An efficient vector system to modify cells genetically. PLoS One 6: e26380, 2011.

20. Schmidt-Wolf IG, Negrin RS, Kiem HP, Blume KG and Weissman IL: Use of a SCID mouse/human lymphoma model to evaluate cytokine-induced killer cells with potent antitumor cell activity. J Exp Med 174: 139-149, 1991.

21. Han H, Ma J, Zhang K, Li W, Liu C, Zhang Y, Zhang G, Ma P, Wang L, Zhang G, et al: Bispecific anti-CD3xanti-HER 2 antibody mediates T cell cytolytic activity to HER2-positive colorectal cancer in vitro and in vivo. Int J Oncol 45: 2446-2454, 2014.
22. Ma J, Han H, Liu D, Li W, Feng H, Xue X, Wu X, Niu G, Zhang G, Zhao Y, et al: HER2 as a promising target for cytotoxicity T cells in human melanoma therapy. PLoS One 8: e73261, 2013.

23. Fu X, Tao L, Rivera A, Williamson S, Song XT, Ahmed N and Zhang X: A simple and sensitive method for measuring tumorspecific T cell cytotoxicity. PLoS One 5: e11867, 2010.

24. Padfield E, Ellis HP and Kurian KM: Current therapeutic advances targeting EGFR and EGFRvIII in glioblastoma. Front Oncol 5: 5, 2015

25. Reusch U, Sundaram M, Davol PA, Olson SD, Davis JB Demel K, Nissim J, Rathore R, Liu PY and Lum LG: Anti-CD3 $\mathrm{x}$ anti-epidermal growth factor receptor (EGFR) bispecific antibody redirects T-cell cytolytic activity to EGFR-positive cancers in vitro and in an animal model. Clin Cancer Res 12: 183-190, 2006.

26. Jäkel CE, Vogt A, Gonzalez-Carmona MA and Schmidt-Wolf IG: Clinical studies applying cytokine-induced killer cells for the treatment of gastrointestinal tumors. J Immunol Res 2014: 897214, 2014.

27. Badr CE and Tannous BA: Bioluminescence imaging: Progress and applications. Trends Biotechnol 29: 624-633, 2011.

28. Nogawa M, Yuasa T, Kimura S, Kuroda J, Sato K, Segawa H, Yokota A and Maekawa T: Monitoring luciferase-labeled cancer cell growth and metastasis in different in vivo models. Cancer Lett 217: 243-253, 2005

29. Tiffen JC, Bailey CG, Ng C, Rasko JE and Holst J: Luciferase expression and bioluminescence does not affect tumor cell growth in vitro or in vivo. Mol Cancer 9: 299, 2010.

30. Patel MA, Kim JE, Ruzevick J, Li G and Lim M: The future of glioblastoma therapy: Synergism of standard of care and immunotherapy. Cancers (Basel) 6: 1953-1985, 2014.

31. Keeren K, Friedrich M, Gebuhr I, Philipp S, Sabat R, Sterry W, Brandt C, Meisel C, Grütz G, Volk HD, et al: Expression of tolerance associated gene-1, a mitochondrial protein inhibiting $\mathrm{T}$ cell activation, can be used to predict response to immune modulating therapies. J Immunol 183: 4077-4087, 2009.

32. Swaika A, Hammond WA and Joseph RW: Current state of anti-PD-L1 and anti-PD-1 agents in cancer therapy. Mol Immunol Mar 4, 2015 (Epub ahead of print). doi: 10.1016/j. molimm.2015.02.009.

33. Suzuki M and Cheung NK: Disialoganglioside GD2 as a therapeutic target for human diseases. Expert Opin Ther Targets 19: 349-362, 2015

34. Zhou Z, Luther N, Ibrahim GM, Hawkins C, Vibhakar R, Handler MH and Souweidane MM: B7-H3, a potential therapeutic target, is expressed in diffuse intrinsic pontine glioma. J Neurooncol 111: 257-264, 2013. 